Bull. Korean Math. Soc. 47 (2010), No. 2, pp. 385-399

DOI 10.4134/BKMS.2010.47.2.385

\title{
ON QUASI-RIGID IDEALS AND RINGS
}

\author{
Chan Yong Hong, Nam Kyun Kim, and Tai Keun Kwak
}

\begin{abstract}
Let $\sigma$ be an endomorphism and $I$ a $\sigma$-ideal of a ring $R$. Pearson and Stephenson called $I$ a $\sigma$-semiprime ideal if whenever $A$ is an ideal of $R$ and $m$ is an integer such that $A \sigma^{t}(A) \subseteq I$ for all $t \geq m$, then $A \subseteq I$, where $\sigma$ is an automorphism, and Hong et al. called $I$ a $\sigma$-rigid ideal if $a \sigma(a) \in I$ implies $a \in I$ for $a \in R$. Notice that $R$ is called a $\sigma$-semiprime ring (resp., a $\sigma$-rigid ring) if the zero ideal of $R$ is a $\sigma$-semiprime ideal (resp., a $\sigma$-rigid ideal). Every $\sigma$-rigid ideal is a $\sigma$-semiprime ideal for an automorphism $\sigma$, but the converse does not hold, in general. We, in this paper, introduce the quasi $\sigma$-rigidness of ideals and rings for an automorphism $\sigma$ which is in between the $\sigma$-rigidness and the $\sigma$-semiprimeness, and study their related properties. A number of connections between the quasi $\sigma$-rigidness of a ring $R$ and one of the Ore extension $R[x ; \sigma, \delta]$ of $R$ are also investigated. In particular, $R$ is a (principally) quasi-Baer ring if and only if $R[x ; \sigma, \delta]$ is a (principally) quasi-Baer ring, when $R$ is a quasi $\sigma$-rigid ring.
\end{abstract}

\section{Definitions}

Let $\sigma$ be an endomorphism of a ring $R$, the additive map $\delta: R \rightarrow R$ is called a $\sigma$-derivation if $\delta(a b)=\delta(a) b+\sigma(a) \delta(b)$ for any $a, b \in R$. For a ring $R$ with an endomorphism $\sigma$ of $R$ and a $\sigma$-derivation $\delta$, the Ore extension $R[x ; \sigma, \delta]$ of $R$ is the ring obtained by giving the polynomial ring over $R$ with new multiplication: $x r=\sigma(r) x+\delta(r)$ for all $r \in R$. If $\delta=0$, we write $R[x ; \sigma]$ for $R[x ; \sigma, 0]$ and it is called the skew polynomial ring (or, an Ore extension of endomorphism type); while $R[[x ; \sigma]]$ is called a skew power series ring.

An endomorphism $\sigma$ of a ring $R$ is called rigid [17] if $a \sigma(a)=0$ implies $a=0$ for $a \in R$. A ring $R$ is called a $\sigma$-rigid ring [9] if there exists a rigid endomorphism $\sigma$ of $R$. The Ore extension $R[x ; \sigma, \delta]$ of $R$ is reduced (i.e., it has no nonzero nilpotent elements) and $\sigma$ is a monomorphism if and only if $R$ is a $\sigma$-rigid ring if and only if $R[x ; \sigma]$ is reduced by [9, Proposition 5] and [10, Proposition 3], respectively. Hence, $\sigma$-rigid rings are reduced rings, but there exists an endomorphism $\sigma$ of a commutative reduced ring which is not a $\sigma$-rigid

Received November 13, 2008.

2000 Mathematics Subject Classification. 16N60, 16S36, 16W60.

Key words and phrases. endomorphism, rigidness, semiprimeness, Ore extension, (principally) quasi-Baer ring. 
ring by [9, Example 9]. An ideal $I$ of $R$ is called a $\sigma$-ideal if $\sigma(I) \subseteq I$. In [13], a $\sigma$-ideal $I$ of a ring $R$ is called a $\sigma$-rigid ideal if $a \sigma(a) \in I$ implies $a \in I$ for $a \in R$, and the connections between $\sigma$-rigid ideals of $R$ and the related ideals of the Ore extension $R[x ; \sigma, \delta]$ of $R$ are investigated. Obviously, $R$ is a $\sigma$-rigid ring if and only if the zero ideal of $R$ is a $\sigma$-rigid ideal. Following [22], for an automorphism $\sigma$ of a ring $R$, a $\sigma$-ideal $I$ of $R$ is called a $\sigma$-semiprime ideal if whenever $A$ is an ideal of $R$ and $m$ is an integer such that $A \sigma^{t}(A) \subseteq I$ for all $t \geq m$, then $A \subseteq I$; the $\operatorname{ring} R$ is called $\sigma$-semiprime if the zero ideal of $R$ is a $\sigma$-semiprime ideal. Notice that $R$ is a $\sigma$-semiprime ring if and only if the skew polynomial ring $R[x ; \sigma]$ is semiprime by [22, Proposition 1.1] (also, [18, Proposition 4.6]). It is well-known that for an automorphism $\sigma$ of a ring $R$, the ring $R$ is $\sigma$-semiprime if and only if whenever $a \in R$ and $m$ is an integer such that $a R \sigma^{t}(a)=0$ for all $t \geq m$, then $a=0$. It is clear that every $\sigma$-rigid ideal (ring) is a $\sigma$-semiprime ideal (ring) for an automorphism $\sigma$. Hence, for an endomorphism $\sigma$ and a $\sigma$-ideal $I$ of a ring $R$, we consider the following condition

$$
a R \sigma(a) \subseteq I \text { implies } a \in I \text { for } a \in R .
$$

Then it can be easily checked that every $\sigma$-rigid ideal satisfies $(*)$ and every $\sigma$-ideal satisfying $(*)$ is $\sigma$-semiprime for an automorphism $\sigma$, but the converses do not hold by the next example, respectively.

Example 1.1. (1) Let $R=\operatorname{Mat}_{2}\left(\mathbb{Z}_{3}\right)$ be the $2 \times 2$ matrix ring over a field $\mathbb{Z}_{3}$. Then $I=\{0\}$ is a maximal (and prime) ideal of $R$. Let $\sigma: R \rightarrow R$ be an automorphism defined by $\sigma\left(\left(\begin{array}{ll}a & b \\ c & d\end{array}\right)\right)=\left(\begin{array}{cc}a & -b \\ -c & d\end{array}\right)$. Suppose that $\left(\begin{array}{ll}a & b \\ c & d\end{array}\right) R \sigma\left(\left(\begin{array}{ll}a & b \\ c & d\end{array}\right)\right) \subseteq I$. Since $R$ is a prime ring, $\left(\begin{array}{ll}a & b \\ c & d\end{array}\right) \in I$ or $\sigma\left(\left(\begin{array}{ll}a & b \\ c & d\end{array}\right)\right) \in I$. Then $\left(\begin{array}{ll}a & b \\ c & d\end{array}\right) \in I$, and therefore $I$ satisfies $(*)$. However, $I$ is not a $\sigma$-rigid ideal by [13, Example 3.3].

(2) Let $\mathbb{Z}_{2}$ be the ring of integers modulo 2 and $R=\mathbb{Z}_{2} \oplus \mathbb{Z}_{2}$. Then $R$ is a commutative reduced ring. Define $\sigma: R \rightarrow R$ by $\sigma(a, b)=(b, a)$. Then the zero ideal $I$ of $R$ does not satisfy $(*)$ : In fact, $(1,0) R \sigma(1,0) \subseteq I$, but $(1,0) \notin I$. We now claim that $R[x ; \sigma]$ is semiprime. Let $f(x) R[x ; \sigma] f(x)=0$, where $f(x)=$ $\sum_{i=0}^{m}\left(a_{i}, b_{i}\right) x^{i} \in R[x ; \sigma]$ with $\left(a_{m}, b_{m}\right) \neq 0$. We may assume that $a_{m} \neq 0$. Then $f(x) x^{t} f(x)=0$ for all integer $t \geq 0$. Thus $\left(a_{m}, b_{m}\right) \sigma^{m+t}\left(a_{m}, b_{m}\right)=0$ of all $t \geq 0$. This implies that $\left(a_{m}, b_{m}\right) \sigma^{m}\left(a_{m}, b_{m}\right)=0$ and $\left(a_{m}, b_{m}\right) \sigma^{m+1}\left(a_{m}, b_{m}\right)$ $=0$. If $m$ is even (or odd), then $\left(a_{m}, b_{m}\right)\left(a_{m}, b_{m}\right)=0$ and $\left(a_{m}, b_{m}\right)\left(b_{m}, a_{m}\right)$ $=0$. Hence $a_{m}=0=b_{m}$. Thus $\left(a_{m}, b_{m}\right)=0$; which is a contradiction. Therefore $R[x ; \sigma]$ is semiprime and so $R$ is $\sigma$-semiprime by [22, Proposition 1.1], equivalently, $I$ is a $\sigma$-semiprime ideal.

Another generalization of $\sigma$-rigid ideals is a $\sigma$-compatible ideal. In [7], an ideal $I$ of a ring $R$ is called a $\sigma$-compatible ideal if for each $a, b \in R, a b \in I \Leftrightarrow$ $a \sigma(b) \in I$. The next example shows that the class of $\sigma$-compatible ideals and the class of $\sigma$-ideals satisfying $(*)$ do not depend on each other.

Example 1.2. (1) In Example 1.1(1), the zero ideal $\{0\}$ of $R$ satisfies $(*)$, but not a $\sigma$-compatible ideal: Indeed, $\left(\begin{array}{ll}1 & 1 \\ 0 & 0\end{array}\right)\left(\begin{array}{ll}2 & 0 \\ 1 & 0\end{array}\right)=0$, but $\left(\begin{array}{ll}1 & 1 \\ 0 & 0\end{array}\right) \sigma\left(\left(\begin{array}{ll}2 & 0 \\ 1 & 0\end{array}\right)\right) \neq 0$. 
(2) Consider a ring $R=\left\{\left(\begin{array}{cc}f(x) & g(x) \\ 0 & f(x)\end{array}\right) \mid f(x), g(x) \in F[x]\right\}$, where $F[x]$ is the polynomial ring over a field $F$. For a nonzero element $a \in F$, let $\sigma$ : $R \rightarrow R$ be an endomorphism defined by $\sigma\left(\left(\begin{array}{cc}f(x) & g(x) \\ 0 & f(x)\end{array}\right)\right)=\left(\begin{array}{cc}f(x) & a g(x) \\ 0 & f(x)\end{array}\right)$. Then the ideal $I=\left\{\left(\begin{array}{cc}0 & g(x) \\ 0 & 0\end{array}\right) \mid g(x) \in\langle p(x)\rangle\right\}$, where $\langle p(x)\rangle$ is an ideal generated by an irreducible polynomial $p(x)$ in $F[x]$, is a $\sigma$-compatible ideal of $R$ by [7, Example 2.5]. However, $I$ does not satisfy $(*)$ : In fact, for $A=\left(\begin{array}{ll}0 & 1 \\ 0 & 0\end{array}\right) \notin I$ we have $A R \sigma(A)=\left\{\left(\begin{array}{ll}0 & 0 \\ 0 & 0\end{array}\right)\right\} \subseteq I$.

Based on these facts, we define the following.

Definition 1.3. Let $\sigma$ be an automorphism of a ring $R$. For a $\sigma$-ideal $I$ of $R, I$ is called a quasi $\sigma$-rigid ideal (or, a strongly $\sigma$-semiprime ideal) of $R$ if $a R \sigma(a) \subseteq I$ implies $a \in I$ for $a \in R$. A ring $R$ is called a quasi $\sigma$-rigid ring (or, a strongly $\sigma$-semiprime ring) if the zero ideal of $R$ is a quasi $\sigma$-rigid ideal.

In this paper, we study both quasi $\sigma$-rigid ideals and quasi $\sigma$-rigid rings for an automorphism $\sigma$. Several relationship between the quasi $\sigma$-rigidness of a ring $R$ and one of the Ore extension $R[x ; \sigma, \delta]$ are also investigated. In particular, we show that $R$ is a (principally) quasi-Baer ring if and only if the Ore extension $R[x ; \sigma, \delta]$ of $R$ is (principally) quasi-Baer when $R$ is a quasi $\sigma$-rigid ring (Theorem 4.3 and Theorem 4.4).

Throughout this paper, $R$ denotes an associative ring with identity. We assume that every endomorphism $\sigma$ of a given ring is an automorphism, unless specified otherwise.

\section{Structures of quasi $\sigma$-rigid ideals}

Recall that for an ideal $I$ of a $\operatorname{ring} R$, the ideal $I$ is called $\sigma$-invariant if $\sigma^{-1}(I)=I$. Note that every $\sigma$-invariant ideal is a $\sigma$-ideal.

Lemma 2.1. Every quasi $\sigma$-rigid ideal of a ring is $\sigma$-invariant and semiprime.

Proof. Let $I$ be a quasi $\sigma$-rigid ideal of a ring $R$. Let $a \in \sigma^{-1}(I)$. Then $\sigma(a) \in I$, and so $a R \sigma(a) \subseteq I$ and hence $a \in I$. Thus $\sigma^{-1}(I) \subseteq I$ and therefore $I$ is $\sigma$-invariant. Now, assume that $a R a \subseteq I$ for $a \in R$. For any $r \in R$, $\operatorname{ar} \sigma(a) R \sigma(\operatorname{ar} \sigma(a)) \subseteq I$ and so $\operatorname{ar} \sigma(a) \in I$. Thus $a \in I$ and therefore $I$ is semiprime.

The converse of Lemma 2.1 does not hold by following.

Example 2.2. Consider the ring $R=\mathbb{Z}_{2} \oplus \mathbb{Z}_{2}$ and the endomorphism $\sigma$ defined by $\sigma(a, b)=(b, a)$, in Example $1.1(2)$. Let $I$ be the prime radical $N_{*}(R)=$ $\{(0,0)\}$ of $R$ (Note that the only proper $\sigma$-ideal is $\{(0,0)\}$ ). Then $I$ is clearly a semiprime ideal and a $\sigma$-invariant ideal, but not a quasi $\sigma$-rigid ideal by Example 1.1(2).

We have the basic equivalences for quasi $\sigma$-rigid ideals as follows. 
Proposition 2.3. Let $I$ be a $\sigma$-ideal of a ring $R$. The following are equivalent to a quasi $\sigma$-rigid ideal $I$ of $R$ :

(1) $\sigma(a) R a \subseteq I$ implies $a \in I$ for any $a \in R$.

(2) For any ideal $A$ of $R, A \sigma(A) \subseteq I$ implies $A \subseteq I$.

(3) For any $\bar{a} \in \bar{R}, \bar{a} \bar{R} \bar{\sigma}(\bar{a})=\overline{0}$ implies $\bar{a}=\overline{0}$, where $\bar{R}=R / I, \bar{a}=a+I$ and $\bar{\sigma}: \bar{R} \rightarrow \bar{R}$ is defined by $\bar{\sigma}(a+I)=\sigma(a)+I$ for $a \in R$.

Proof. (1) Suppose that $I$ is a quasi $\sigma$-rigid ideal. Then $I$ is semiprime by Lemma 2.1. If $\sigma(a) R a \subseteq I$ for $a \in R$, then $a R \sigma(a) \subseteq I$ and thus $a \in I$. Conversely, assume that $\sigma(a) R a \subseteq I$ implies $a \in I$ for any $a \in R$. If $b R b \subseteq$ $I$, then $\sigma(\sigma(b) r b) R \sigma(b) r b=\sigma^{2}(b) \sigma(r) \sigma(b R b) r b \subseteq I$ for any $r \in R$, and so $\sigma(b) r b \in I$ and thus $b \in I$ by the assumption, entailing that $I$ is semiprime. Hence, $a R \sigma(a) \subseteq I$ implies $\sigma(a) R a \subseteq I$, and so $a \in I$ by the assumption, concluding that $I$ is a quasi $\sigma$-rigid ideal. (2) Let $I$ be a quasi $\sigma$-rigid ideal and $a \in A$. If $A \sigma(A) \subseteq I$, then $a R \sigma(a) \subseteq I$, and so $a \in I$. Consequently, $A \subseteq I$. Conversely, assume that for any ideal $A$ of $R, A \sigma(A) \subseteq I$ implies $A \subseteq I$. If $a R \sigma(a) \subseteq I$, then $\operatorname{RaR} \sigma(\operatorname{RaR})=\operatorname{RaR} \sigma(a) R \subseteq I$ and so $R a R \subseteq I$, and hence $a \in I$, implying that $I$ is a quasi $\sigma$-rigid ideal. (3) is obvious.

For the remainder of this paper, let $\delta$ be a $\sigma$-derivation of a ring $R$. Recall that an ideal $I$ of $R$ is called a $\delta$-ideal if $\delta(I) \subseteq I$.

Lemma 2.4. Let $I$ be a quasi $\sigma$-rigid ideal of a ring $R$.

(1) If $a R b \subseteq I$ for $a, b \in R$, then $a R \sigma^{n}(b), \sigma^{n}(a) R b \subseteq I$ for every positive integer $n$. Conversely, if $a R \sigma^{k}(b)$ or $\sigma^{k}(a) R b \subseteq I$ for some positive integer $k$, then $a R b \subseteq I$.

(2) If $I$ is a $\delta$-ideal with $a R b \subseteq I$ for $a, b \in R$, then $a R \delta^{n}(b), \delta^{n}(a) R b \subseteq I$ for every positive integer $n$.

Proof. We freely use the fact that every quasi $\sigma$-rigid ideal is $\sigma$-invariant and semiprime by Lemma 2.1. (1) Suppose that $a R b \subseteq I$ for $a, b \in R$. It is enough to show that $a R \sigma(b), \sigma(a) R b \subseteq I$. For any $r \in R, \operatorname{br} \sigma(a) R \sigma(b r \sigma(a))=$ $\operatorname{br} \sigma(a R b) \sigma(r \sigma(a)) \subseteq I$. Since $I$ is a quasi $\sigma$-rigid ideal, $b r \sigma(a) \in I$ and so $b R \sigma(a) \subseteq I$. Hence, $\sigma(a) R b \subseteq I$ since $I$ is a semiprime ideal. Next, we obtain $b R a \subseteq I$ since $a R b \subseteq I$ and $I$ is a semiprime ideal. By the same method, we get $a R \sigma(b) \subseteq I$. Conversely, suppose that $a R \sigma^{k}(b) \subseteq I$ for some positive integer $k$. Then, by the above arguments, $\sigma^{k}(a R b)=\sigma^{k}(a) R \sigma^{k}(b) \subseteq I$. Since $I$ is a $\sigma$-invariant ideal, $\sigma^{k-1}(a R b) \subseteq \sigma^{-1}(I)=I$. Continuing this process, we have $a R b \subseteq I$. Similarly, $\sigma^{k}(a) R b \subseteq I$ for some positive integer $k$ implies $a R b \subseteq I$. (2) Assume that $I$ is a $\delta$-ideal and $a R b \subseteq I$ for $a, b \in R$. It is sufficient to show that $a R \delta(b), \delta(a) R b \subseteq I$. Let $a R b \subseteq I$. Note that $b R a \subseteq I$ and so $b R \sigma(a) \subseteq I$ by (1). For any $r \in R, \delta($ arb $)=\sigma(a r) \delta(b)+\delta(a r) b \in I$. Thus $(\sigma(a r) \delta(b) R)^{2}=\delta(a r b) R \sigma(a r) \delta(b) R-\delta(a r) b R \sigma(a r) \delta(b) R \subseteq I$ because $\delta(a r b) \in I$ and $b R \sigma(a) \subseteq I$. Since $I$ is semiprime, we have $\sigma(a) R \delta(b) \subseteq I$ and so $a R \delta(b) \subseteq I$ by (1). Similarly, $b R a \subseteq I$ from $a R b \subseteq I$ implies $\delta(a) R b \subseteq I$, completing proof. 
Corollary 2.5. Let $I$ be a $\sigma$-ideal of a ring $R$. The ideal $I$ is a quasi $\sigma$-rigid ideal if and only if $I$ is a semiprime ideal, and $a R \sigma(b) \subseteq I \Leftrightarrow a R b \subseteq I$ for $a, b \in R$.

Proof. It follows from Lemma 2.1 and Lemma 2.4.

Theorem 2.6. Let $I$ be a quasi $\sigma$-rigid ideal of a ring $R$.

(1) Let $p(x)=\sum_{i=0}^{m} a_{i} x^{i}$ and $q(x)=\sum_{j=0}^{n} b_{j} x^{j} \in R[x ; \sigma, \delta]$. If I is a $\delta$-ideal of $R$, then $p(x) R[x ; \sigma, \delta] q(x) \subseteq I[x ; \sigma, \delta]$ if and only if $a_{i} R b_{j} \subseteq I$ for all $i$ and $j$.

(2) Let $p(x)=\sum_{i=0}^{\infty} a_{i} x^{i}$ and $q(x)=\sum_{j=0}^{\infty} b_{j} x^{j} \in R[[x ; \sigma]]$. Then

$p(x) R[[x ; \sigma]] q(x) \subseteq I[[x ; \sigma]]$ if and only if $a_{i} R b_{j} \subseteq I$ for all $i$ and $j$.

Proof. (1) Suppose that $p(x) R[x ; \sigma, \delta] q(x) \subseteq I[x ; \sigma, \delta]$. Then $p(x) r q(x) \in$ $I[x ; \sigma, \delta]$ for any $r \in R$, and put

$$
\left(\sum_{i=0}^{m} a_{i} x^{i}\right) r\left(\sum_{j=0}^{n} b_{j} x^{j}\right)=c_{m+n} x^{m+n}+c_{m+n-1} x^{m+n-1}+\cdots+c_{1} x+c_{0} \in I[x ; \sigma, \delta] .
$$

We claim that $a_{i} R b_{j} \subseteq I$ for all $0 \leq i \leq m$ and $0 \leq j \leq n$. We proceed by induction on $i+j$. When $i+j=m+n$, we have $c_{m+n}=a_{m} \sigma^{m}(r) \sigma^{m}\left(b_{n}\right) \in I$ by above, and so $a_{m} R b_{n} \subseteq I$ by Lemma 2.4. Now suppose that our claim is true for $i+j>k \geq 0$. Consider

$$
c_{k}=\sum_{i+j=k} a_{i} \sigma^{i}(r) \sigma^{i}\left(b_{j}\right)+\sum_{i+j>k} a_{i} R \sigma^{i_{1}} \delta^{j_{1}} \sigma^{i_{2}} \delta^{j_{2}} \cdots \sigma^{i_{l}} \delta^{j_{l}}\left(b_{j}\right) \subseteq I
$$

for any $r \in R$ and some nonnegative integers $i_{1}, \ldots, i_{l}, j_{1}, \ldots, j_{l}$. Since

$$
\sum_{i+j>k} a_{i} R \sigma^{i_{1}} \delta^{j_{1}} \sigma^{i_{2}} \delta^{j_{2}} \cdots \sigma^{i_{l}} \delta^{j_{l}}\left(b_{j}\right) \subseteq I,
$$

by induction hypothesis and Lemma 2.4 , we obtain

$$
c_{k}=\sum_{i+j=k} a_{i} \sigma^{i}(r) \sigma^{i}\left(b_{j}\right) \in I
$$

for any $r \in R$. Multiplying Eq.(1) by $R a_{k}$ from the right hand-side, we have

$$
\left(\sum_{i+j=k} a_{i} \sigma^{i}(r) \sigma^{i}\left(b_{j}\right)\right) R a_{k}=a_{k} \sigma^{k}(r) \sigma^{k}\left(b_{0}\right) R a_{k} \subseteq I
$$

since $\sigma^{u}\left(b_{j}\right) R a_{i} \subseteq I$ for all $i+j>k$ and any nonnegative integer $u$, by induction hypothesis and Lemma 2.4(1). Then for any $r \in R,\left(a_{k} \sigma^{k}(r) \sigma^{k}\left(b_{0}\right) R\right)^{2} \subseteq I$ and so $a_{k} \sigma^{k}(r) \sigma^{k}\left(b_{0}\right) \in I$ by Lemma 2.1. Hence $a_{k} R \sigma^{k}\left(b_{0}\right) \subseteq I$ and so $a_{k} R b_{0} \subseteq I$ by Lemma 2.4(1). Thus Eq.(1) becomes

$$
\sum_{i+j=k, 0 \leq i \leq k-1} a_{i} \sigma^{i}(r) \sigma^{i}\left(b_{j}\right) \in I .
$$


Multiplying Eq.(2) by $R a_{k-1}$ from the right hand-side,

$$
a_{k-1} \sigma^{k-1}(r) \sigma^{k-1}\left(b_{1}\right) R a_{k-1} \subseteq I
$$

and so $a_{k-1} R b_{1} \subseteq I$ by the same method above. Continuing in this manner, we obtain that $a_{i} R b_{j} \subseteq I$ for all $i, j$ with $i+j=k$. Therefore $a_{i} R b_{j} \subseteq I$ for all $0 \leq i \leq m$ and $0 \leq j \leq n$. The converse follows directly from Lemma 2.4.

(2) Suppose that $\bar{p}(x) R[[x ; \sigma]] q(x) \subseteq I[[x ; \sigma]]$. Then $p(x) r q(x) \in I[[x ; \sigma]]$ for any $r \in R$. Then

$$
\left(\sum_{i=0}^{\infty} a_{i} x^{i}\right) r\left(\sum_{j=0}^{\infty} b_{j} x^{j}\right)=\sum_{k=0}^{\infty}\left(\sum_{i+j=k} a_{i} \sigma^{i}(r) \sigma^{i}\left(b_{j}\right) x^{i+j}\right) \in I[[x ; \sigma]] .
$$

We claim that $a_{i} R b_{j} \subseteq I$ for all $i, j$. We proceed by induction on $i+j$. For $i+j=0$, we have $a_{0} R b_{0} \subseteq I$ by Eq.(3). Now assume that our claim is true for $i+j \leq n-1$. Note that $a_{i} R \sigma^{u}\left(b_{j}\right)$ and $\sigma^{v}\left(b_{j}\right) R a_{i} \subseteq I$ for any nonnegative integers $u$ and $v$, by induction hypothesis and Lemma 2.4(1), when $i+j \leq n-1$. We show that $a_{i} R b_{j} \in I$ for $i+j=n$. From Eq.(3), we have

$$
\sum_{i+j=n} a_{i} \sigma^{i}(r) \sigma^{i}\left(b_{j}\right) \in I .
$$

Multiplying Eq.(4) by $R a_{0}$ from the right hand-side, we obtain $\left(a_{0} r b_{n}\right) R a_{0} \subseteq I$, and so $a_{0} r b_{n} \in I$ and thus $a_{0} R b_{n} \subseteq I$. Thus Eq.(4) becomes

$$
\sum_{i+j=n, 1 \leq i \leq n} a_{i} \sigma^{i}(r) \sigma^{i}\left(b_{j}\right) \in I .
$$

Multiplying Eq.(5) by $R a_{1}$ from the right hand-side, we obtain $a_{1} R b_{n-1} \subseteq I$ by the similar arguments as above. Continuing this process, we can prove that $a_{i} R b_{j} \subseteq I$ for all $i, j$ with $i+j=n$. Therefore, $a_{i} R b_{j} \subseteq I$ for all $i$ and $j$. The converse also follows directly from Lemma 2.4(1).

Corollary 2.7. Let $I$ be a quasi $\sigma$-rigid ideal of a ring $R$.

(1) $I[x ; \sigma, \delta]$ is a semiprime ideal of $R[x ; \sigma, \delta]$, when $I$ is a $\delta$-ideal of $R$.

(2) $I[[x ; \sigma]]$ is a semiprime ideal of $R[[x ; \sigma]]$.

Proof. (1) Let $p(x) R[x ; \sigma, \delta] p(x) \subseteq I[x ; \sigma, \delta]$, where $p(x)=\sum_{i=0}^{m} a_{i} x^{i} \in R[x ; \sigma, \delta]$. Then $a_{i} R a_{i} \subseteq I$ for all $0 \leq i \leq m$ by Theorem 2.6. Since $I$ is a semiprime ideal of $R$ by Lemma 2.1, we have $a_{i} \in I$ for all $0 \leq i \leq m$, and thus $p(x) \in I[x ; \sigma, \delta]$. Therefore $I[x ; \sigma, \delta]$ is a semiprime ideal of $\bar{R}[x ; \sigma, \delta]$. (2) By the same method as (1).

Recall from [21], a one-sided ideal $I$ of a $\operatorname{ring} R$ has the insertion of factors property (or simply, IFP) if $a b \in I$ implies $a R b \subseteq I$ for $a, b \in R$.

Lemma 2.8. For an ideal $I$ of a ring $R, I$ is a quasi $\sigma$-rigid ideal and has the IFP if and only if $I$ is a $\sigma$-rigid ideal. 
Proof. Let $I$ be a quasi $\sigma$-rigid ideal and have the IFP. If $a \sigma(a) \in I$ for $a \in R$, then $a R \sigma(a) \subseteq I$ by the IFP, and hence $a \in I$, concluding that $I$ is a $\sigma$-rigid ideal. Conversely, suppose that $I$ is a $\sigma$-rigid ideal. Then $I$ is a completely semiprime ideal (i.e., $a^{2} \in I$ implies $a \in I$ for $a \in R$ ) of $R$ by [13, Proposition 2.2(1)], and so $I$ has the IFP by [21, Lemma 3.2(a)].

Let $N(R), N_{*}(R)$ and $N^{*}(R)$ denote the set of all nilpotent elements, the prime radical and the upper nilradical (i.e., the sum of all nil ideals) of a ring $R$, respectively. A ring $R$ is called 2-primal [3] if $N_{*}(R)=N(R)$, and a ring $R$ is called $N I$ [20] if $N^{*}(R)=N(R)$. It is well-known that a ring $R$ is 2-primal if and only if $N_{*}(R)$ is a completely semiprime ideal of $R$, and a $\operatorname{ring} R$ is NI if and only if $N^{*}(R)$ is a completely semiprime ideal of $R$. Every 2-primal ring is NI, but the converse does not hold in general.

We use $R[x]$ to denote the polynomial ring with an indeterminate $x$ over a ring $R$.

Theorem 2.9. (1) $R$ is a 2-primal ring and $N_{*}(R)$ is a quasi $\sigma$-rigid ideal if and only if $N_{*}(R)$ is a $\sigma$-rigid ideal of $R$. In particular, if $R$ is a 2-primal ring, then $f(x) g(x) \in N(R)[x]$ if and only if $a_{i} b_{j} \in N(R)$ for all $i$ and $j$, where $f(x)=\sum_{i=0}^{m} a_{i} x^{i}$ and $g(x)=\sum_{j=0}^{n} b_{j} x^{j} \in R[x]$.

(2) $R$ is an $N I$ ring and $N^{*}(R)$ is a quasi $\sigma$-rigid ideal if and only if $N^{*}(R)$ is a $\sigma$-rigid ideal of $R$.

Proof. (1) Note that $R$ is a 2-primal ring if and only if $N_{*}(R)$ has the IFP by [16, Theorem 2.1]. Hence, by Lemma $2.8, N_{*}(R)$ is a quasi $\sigma$-rigid ideal if and only if $N_{*}(R)$ is a $\sigma$-rigid ideal, when $R$ is a 2-primal ring. Now, let $R$ be a 2-primal ring and $f(x)=\sum_{i=0}^{m} a_{i} x^{i}$ and $g(x)=\sum_{j=0}^{n} b_{j} x^{j} \in R[x]$. It is well-known that the polynomial ring $R[x]$ over $R$ is 2-primal by [3, Proposition 2.6]. Assume that $f(x) g(x) \in N(R)[x]$. Then $f(x) g(x) \in N(R)[x]=N(R[x])=N_{*}(R[x])$ if and only if $f(x) R[x] g(x) \subseteq N(R)[x]=N_{*}(R[x])$ by [3, Proposition 2.6] and [16, Theorem 2.1] if and only if $a_{i} R b_{j} \subseteq N(R)$ for all $i$ and $j$ by Theorem 2.6(1) if and only if $a_{i} b_{j} \in N(R)$ by [16, Theorem 2.1]. (2) It follows from the fact that $N^{*}(R)$ has the IFP if and only if $R$ is an NI ring by [12, Theorem 8].

Let $\rho(R)$ be either $N_{*}(R)$ or $N^{*}(R)$, and put

$$
\Gamma(R)= \begin{cases}\operatorname{mSpec}(R), & \text { if } \rho(R)=\mathbf{N}_{*}(R) \\ \operatorname{mSpec}_{\mathbf{S}}(R), & \text { if } \rho(R)=\mathbf{N}^{*}(R),\end{cases}
$$

where $\operatorname{mSpec}(R)$ and $\mathbf{m S p e c}_{\mathbf{S}}(R)$ denote the set of all minimal prime ideal and all minimal strongly prime ideals of $R$, respectively.

Corollary 2.10. Assume that $\rho(R)$ is a completely semiprime ideal of a ring $R$. The following are equivalent:

(1) $\rho(R)$ is a quasi $\sigma$-rigid ideal.

(2) $\rho(R)$ is a $\sigma$-rigid ideal.

(3) $P$ is $\sigma$-invariant for each $P \in \Gamma(R)$. 
(4) $\sigma^{-1}(P) \subseteq P$ for each $P \in \Gamma(R)$.

(5) $P$ is a $\sigma$-ideal for each $P \in \Gamma(R)$.

Proof. It follows from Theorem 2.9 and [13, Proposition 3.4].

\section{Extensions of quasi $\sigma$-rigid rings}

Recall that a ring $R$ is called quasi $\sigma$-rigid if the zero ideal of $R$ is a quasi $\sigma$-rigid ideal. For an automorphism $\sigma$, every $\sigma$-rigid ring is a quasi $\sigma$-rigid ring and every quasi $\sigma$-rigid ring is a $\sigma$-semiprime ring, but the converses do not hold by Example 1.1, respectively. Every quasi $\sigma$-rigid ring is a semiprime ring by Lemma 2.1 (but not reduced by Example 1.1(1)). There exists a semiprime ring $R$ with an endomorphism $\sigma$ such that the skew polynomial $\operatorname{ring} R[x ; \sigma]$ is not semiprime [14, Example 4.3]. However, we have the following result by Corollary 2.7.

Corollary 3.1. If $R$ is a quasi $\sigma$-rigid ring, then $R[x ; \sigma, \delta]$ is a semiprime ring.

It can be easily checked that any prime ring with an automorphism $\sigma$ is a quasi $\sigma$-rigid ring; while for the ring $R=\mathbb{Z}_{2} \oplus \mathbb{Z}_{2}$ with an automorphism $\sigma$ in Example 1.1(2), both $R$ and $R[x ; \sigma]$ are semiprime rings, but $R$ is not quasi $\sigma$-rigid. Note that the minimal prime ideal $\mathbb{Z}_{2} \oplus\{0\}$ of $R$ is not a $\sigma$-ideal. However,

Proposition 3.2. If $R$ is a semiprime ring whose minimal prime ideals are $\sigma$-ideals, then $R$ is a quasi $\sigma$-rigid ring.

Proof. Suppose $a R \sigma(a)=0$ for $a \in R$. Then $a R \sigma(a) \subseteq P$ for any minimal prime ideal $P$ of $R$. So $a \in P$ or $\sigma(a) \in P$. Since $P$ is a $\sigma$-ideal, we get $\sigma(a) \in P$ and so $\sigma(a) \in N_{*}(R)=0$ because $R$ is semiprime. Thus $a=0$, concluding that $R$ is a quasi $\sigma$-rigid ring.

Recall that $R$ is called a $\sigma$-compatible ring [1] (or [8]) if for each $a, b \in R$, $a b=0 \Leftrightarrow a \sigma(b)=0$, equivalently, the zero ideal of $R$ is a $\sigma$-compatible ideal. Every $\sigma$-rigid ring is a $\sigma$-compatible ring, but the converse does not hold, in general. Note that the class of quasi $\sigma$-rigid rings and the class of $\sigma$-compatible rings do not depend on each other by Example 1.2(1) and the following example.

Example 3.3. We consider a ring $R=\left\{\left(\begin{array}{ll}a & t \\ 0 & a\end{array}\right) \mid a \in \mathbb{Z}, t \in \mathbb{Q}\right\}$, where $\mathbb{Z}$ and $\mathbb{Q}$ are the set of all integers and all rational numbers, respectively. Let $\sigma: R \rightarrow R$ be an endomorphism defined by $\sigma\left(\left(\begin{array}{cc}a & t \\ 0 & a\end{array}\right)\right)=\left(\begin{array}{cc}a & t / 2 \\ 0 & a\end{array}\right)$. Note that for $\left(\begin{array}{ll}0 & 1 \\ 0 & 0\end{array}\right) \neq 0$, we have $\left(\begin{array}{ll}0 & 1 \\ 0 & 0\end{array}\right)\left(\begin{array}{ll}a & t \\ 0 & a\end{array}\right) \sigma\left(\left(\begin{array}{ll}0 & 1 \\ 0 & 0\end{array}\right)\right)=0$ for any $a \in \mathbb{Z}$ and $t \in \mathbb{Q}$. This yields that $R$ is not a quasi $\sigma$-rigid ring. Now, we show that $R$ is a $\sigma$-compatible ring. Suppose that $A B=0$ for $A=\left(\begin{array}{ll}a & t \\ 0 & a\end{array}\right), B=\left(\begin{array}{ll}b & s \\ 0 & b\end{array}\right) \in R$. Then $a b=0$ and $a s+t b=0$, and so $a=0$ or $b=0$. If $a=0$, then $t=0$ and hence $A=0$. Similarly, if $b=0$, then $s=0$ and so $B=0$, entailing that $A B=0 \Leftrightarrow A \sigma(B)=0$. Therefore $R$ is a $\sigma$-compatible ring. 
A ring $R$ is called semicommutative if $a b=0$ implies $a R b=0$ for $a, b \in R$, which every reduced ring is semicommutative. Notice that a ring is semicommutative if and only if the zero ideal has the IFP, and so semicommutative rings are also called IFP-rings. Recently, the concept of the semicommutativity of a ring is extended to an endomorphism of a ring. An endomorphism $\sigma$ of a ring $R$ is called semicommutative [2, Definition 2.1] if whenever $a b=0$ for $a, b \in R, a R \sigma(b)=0$; a ring $R$ is called $\sigma$-semicommutative if there exists a semicommutative endomorphism $\sigma$ of $R$. The semicommutativity and the $\sigma$-semicommutativity of a ring are independent each other by [2, Example 2.3 and Example 2.7]. In a semicommutative ring, the quasi $\sigma$-rigidness and the $\sigma$ rigidness of a ring coincide by Lemma 2.8. Moreover, for a $\sigma$-semicommutative ring we have the following:

Proposition 3.4. Let $R$ be a $\sigma$-semicommutative ring. The following are equivalent:

(1) $R$ is a $\sigma$-rigid ring.

(2) $R$ is a quasi $\sigma$-rigid ring.

(3) $R$ is a $\sigma$-semiprime ring.

Proof. It is enough to show that $(3) \Rightarrow(1)$. Assume that $R$ is a $\sigma$-semiprime ring. Let $a \sigma(a)=0$ for $a \in R$. Then $a R \sigma^{t}(a)=0$ for any positive integer $t$ by [2, Remark 2.2]. Thus $a=0$ and therefore $R$ is a $\sigma$-rigid ring.

Note that the ring $R$, in Example 3.3, is a $\sigma$-semicommutative ring by the same method as in [2, Example 2.5(1)]. Hence, any condition in Proposition 3.4 cannot be replaced by " $R$ is a $\sigma$-compatible ring".

Corollary 3.5. If $R$ is a semiprime and semicommutative ring, then $R$ is a reduced ring.

For an automorphism $\sigma$ of a ring $R$, the map $\bar{\sigma}: R[x] \rightarrow R[x]$ defined by $\bar{\sigma}\left(\sum_{i=0}^{m} a_{i} x^{i}\right)=\sum_{i=0}^{m} \sigma\left(a_{i}\right) x^{i}$ is an automorphism of the polynomial ring $R[x]$ and clearly this map extends $\sigma$. The ring of Laurent polynomials in $x$, coefficients in a ring $R$, consists of all formal sums $\sum_{i=k}^{n} m_{i} x^{i}$ with obvious addition and multiplication, where $m_{i} \in R$ and $k, n$ are (possibly negative) integers; denote it by $R\left[x ; x^{-1}\right]$. The map $\bar{\sigma}: R\left[x, x^{-1}\right] \rightarrow R\left[x, x^{-1}\right]$ defined by $\bar{\sigma}\left(\sum_{i=k}^{n} a_{i} x^{i}\right)=\sum_{i=k}^{n} \sigma\left(a_{i}\right) x^{i}$ extends $\sigma$ and is also an automorphism of $R\left[x, x^{-1}\right]$.

Proposition 3.6. For a ring $R$, the following are equivalent:

(1) $R$ is a quasi $\sigma$-rigid ring.

(2) $R[x]$ is a quasi $\bar{\sigma}$-rigid ring.

(3) $R\left[x, x^{-1}\right]$ is a quasi $\bar{\sigma}$-rigid ring.

Proof. $(1) \Leftrightarrow(2)$ Let $R$ be a quasi $\sigma$-rigid ring. Suppose that $R[x]$ is not a quasi $\bar{\sigma}$-rigid ring. Then there exists a nonzero polynomial $f(x)=a_{0}+a_{1} x+\cdots+a_{n} x^{n}$ such that $f(x) R[x] \bar{\sigma}(f(x))=0$. We may assume that $a_{n} \neq 0$. By simple 
computation, we have $a_{n} R \sigma\left(a_{n}\right)=0$. Since $R$ is a quasi $\sigma$-rigid ring, we obtain $a_{n}=0$; which is a contradiction. Conversely, assume (2) and let $a R \sigma(a)=0$ for $a \in R$. Note that $a R[x] \bar{\sigma}(a)=0$. Since $R[x]$ is a quasi $\bar{\sigma}$-rigid ring, we have $a=0$. Hence, $R$ is a quasi $\sigma$-rigid ring. (1) $\Leftrightarrow(3)$ can be proved by the similar arguments above.

Note that $I$ is a quasi $\sigma$-rigid ideal of a ring $R$ if and only if the factor ring $R / I$ is a quasi $\bar{\sigma}$-rigid ring by Proposition 2.3, where $\bar{\sigma}: R / I \rightarrow R / I$ is defined by $\bar{\sigma}(a+I)=\sigma(a)+I$ for $a \in R$. The following example shows that there exists a ring $R$ with an automorphism $\sigma$ such that for any nonzero proper ideal $I$ of $R, I$ is a $\sigma$-rigid ideal and so $R / I$ is a quasi $\bar{\sigma}$-rigid ring, but $R$ is not a quasi $\sigma$-rigid ring. Moreover, the next example illuminates that the subring of a quasi $\sigma$-rigid ring need not be a quasi $\sigma$-rigid ring, combining with Example 1.1(1).

Example 3.7. Let $R=\left(\begin{array}{cc}F & F \\ 0 & F\end{array}\right)$ where $F$ is a field, and $\sigma$ be an endomorphism of $R$ defined by $\sigma\left(\left(\begin{array}{ll}a & b \\ 0 & c\end{array}\right)\right)=\left(\begin{array}{cc}a & -b \\ 0 & c\end{array}\right)$. For $a=\left(\begin{array}{ll}0 & 1 \\ 0 & 0\end{array}\right) \in R, a R \sigma(a)=0$, but $a \neq 0$, and so $R$ is not a quasi $\sigma$-rigid ring. For the only nonzero proper ideals $I=\left(\begin{array}{cc}F & F \\ 0 & 0\end{array}\right), J=\left(\begin{array}{ll}0 & F \\ 0 & F\end{array}\right)$ and $K=\left(\begin{array}{ll}0 & F \\ 0 & 0\end{array}\right)$ of $R$, it can be easily checked that $I, J$ and $K$ are $\sigma$-rigid ideals, thus $R / I, R / J$ and $R / K$ are quasi $\bar{\sigma}$-rigid rings by Proposition 2.3.

For an automorphism $\sigma$ of a ring $R$, the map $\bar{\sigma}: \operatorname{Mat}_{n}(R) \rightarrow \operatorname{Mat}_{n}(R)$ defined by $\bar{\sigma}\left(\left(a_{i j}\right)\right)=\left(\sigma\left(a_{i j}\right)\right)$ is an automorphism of the $n \times n$ full matrix ring $\operatorname{Mat}_{n}(R)$.

Theorem 3.8. For a ring $R$, the following are equivalent:

(1) $R$ is a quasi $\sigma$-rigid ring.

(2) $\operatorname{Mat}_{n}(R)$ is a quasi $\bar{\sigma}$-rigid ring for any $n \geq 2$.

(3) $\operatorname{Mat}_{n}(R)$ is a quasi $\bar{\sigma}$-rigid ring for some $n \geq 2$.

Proof. $(1) \Rightarrow(2)$ Let $R$ be a quasi $\sigma$-rigid ring and $n \geq 2$. Suppose that $A \operatorname{Mat}_{n}(R) \bar{\sigma}(A)=0$ for $A=\left(a_{i j}\right) \in \operatorname{Mat}_{n}(R)$. Let $E_{i j}$ denote the matrix unit in $\operatorname{Mat}_{n}(R)$ with $(i, j)$-entry 1 and zero elsewhere. Then $A\left(r E_{i j}\right) \bar{\sigma}(A)=0$ implies $a_{i j} r \sigma\left(a_{i j}\right)=0$ for each $i, j \in\{1,2, \ldots, n\}$ and any $r \in R$. Hence, $a_{i j} R \sigma\left(a_{i j}\right)=0$, entailing $a_{i j}=0$ since $R$ is a quasi $\sigma$-rigid ring. Therefore $A=0$, concluding that $\operatorname{Mat}_{n}(R)$ is a quasi $\bar{\sigma}$-rigid ring. $(2) \Rightarrow(3)$ is obvious. $(3) \Rightarrow(1)$ Assume that $\operatorname{Mat}_{n}(R)$ is a quasi $\bar{\sigma}$-rigid ring for $n \geq 2$. Let $a R \sigma(a)=0$ for $a \in R$ and $A=a \sum_{i=1}^{n} E_{i i}$ in $\operatorname{Mat}_{n}(R)$. Then $A_{M a t}(R) \bar{\sigma}(A)=0$. Since $\operatorname{Mat}_{n}(R)$ is a quasi $\bar{\sigma}$-rigid ring, we get $A=0$, and hence $a=0$, proving that $R$ is a quasi $\sigma$-rigid ring.

From Theorem 3.8, one may conjecture that the $n \times n$ upper triangular matrix ring $U_{n}(R)$ over a quasi $\sigma$-rigid ring $R$ is quasi $\bar{\sigma}$-rigid for $n \geq 2$, but the possibility is erased by the following.

Example 3.9. Let $R$ be a ring with any endomorphism $\sigma$. Let $A=E_{1 n} \in$ $U_{n}(R)$, where $E_{i j}$ is the matrix unit in $U_{n}(R)$. Then $A U_{n}(R) \bar{\sigma}(A)=0$ (regardless of $\sigma)$. Thus the $n \times n$ upper triangular matrix $\operatorname{ring} U_{n}(R)$ over $R$ is 
not a quasi $\bar{\sigma}$-rigid ring for any $n \geq 2$. Moreover, for a ring $R$ and $n \geq 2$, let

$$
\begin{gathered}
S_{n}(R)=\left\{\left(\left(\begin{array}{ccccc}
a & a_{12} & a_{13} & \cdots & a_{1 n} \\
0 & a & a_{23} & \cdots & a_{2 n} \\
0 & 0 & a & \cdots & a_{3 n} \\
\vdots & \vdots & \vdots & \ddots & \vdots \\
0 & 0 & 0 & \cdots & a
\end{array}\right) \mid a, a_{i j} \in R\right\}\right. \text { and } \\
V_{n}(R)=\left\{\left(\begin{array}{cccccc}
a_{1} & a_{2} & a_{3} & a_{4} & \cdots & a_{n} \\
0 & a_{1} & a_{2} & a_{3} & \cdots & a_{n-1} \\
0 & 0 & a_{1} & a_{2} & \cdots & a_{n-2} \\
\vdots & \vdots & \vdots & \vdots & \cdots & \vdots \\
0 & 0 & 0 & 0 & \cdots & a_{2} \\
0 & 0 & 0 & 0 & \cdots & a_{1}
\end{array}\right) \mid a_{1}, a_{2}, \ldots, a_{n} \in R\right\} .
\end{gathered}
$$

By the same method, we conclude that $S_{n}(R)$ and $V_{n}(R)$ are not a quasi $\bar{\sigma}$-rigid ring for any $n \geq 2$. Since $V_{n}(R) \cong R[x] /\left\langle x^{n}\right\rangle$ by [19] where $\left\langle x^{n}\right\rangle$ is an ideal of $R[x]$ generated by $x^{n}, R[x] /\left\langle x^{n}\right\rangle$ is not a quasi $\bar{\sigma}$-rigid ring for $n \geq 2$ either, where $\bar{\sigma}\left(f(x)+\left\langle x^{n}\right\rangle\right)=\sigma(f(x))+\left\langle x^{n}\right\rangle$ for $f(x) \in R[x]$.

For an automorphism $\sigma$ and an idempotent $e$ of a $\operatorname{ring} R$ such that $\sigma(e)=e$, the map $\bar{\sigma}: e R e \rightarrow e R e$ defined by $\bar{\sigma}(e r e)=e \sigma(r) e$ is an automorphism of $e R e$.

Proposition 3.10. For a ring $R$, assume that $\sigma(e)=e$ for $e^{2}=e \in R$. If $R$ is a quasi $\sigma$-rigid ring, then eRe is a quasi $\bar{\sigma}$-rigid ring.

Proof. For eae $\in e R e$, suppose that eae $(e R e) \bar{\sigma}(e a e)=0$. Then

$$
0=e a e(e R e) \bar{\sigma}(e a e)=e a e(e R e) e \sigma(a) e=(e a e) R \sigma(e a e) .
$$

Since $R$ is a quasi $\sigma$-rigid ring, eae $=0$ and so $e R e$ is a quasi $\bar{\sigma}$-rigid ring.

The condition " $\sigma(e)=e$ for $e^{2}=e \in R$ " in Proposition 3.10 cannot be dropped by the following example.

Example 3.11. Consider the quasi $\sigma$-rigid $\operatorname{ring} R=\operatorname{Mat}_{2}\left(\mathbb{Z}_{3}\right)$ where $\sigma$ is defined by $\sigma\left(\left(\begin{array}{ll}a & b \\ c & d\end{array}\right)\right)=\left(\begin{array}{cc}a & -b \\ -c & d\end{array}\right)$ as in Example 1.1(1). For an idempotent $e=\left(\begin{array}{ll}0 & 1 \\ 0 & 1\end{array}\right) \in R, \sigma(e) \neq e$. Let $a=\left(\begin{array}{ll}0 & 0 \\ 1 & 1\end{array}\right) \in R$. Then $e a e=\left(\begin{array}{ll}0 & 2 \\ 0 & 2\end{array}\right) \neq 0$. But for any $r=\left(\begin{array}{ll}s & t \\ u & v\end{array}\right) \in R$, eae $($ ere $) \bar{\sigma}($ eae $)=\operatorname{eaere} \sigma(a) e=0$, and so eae $(e R e) \bar{\sigma}(e a e)=0$, implying that $e R e$ is not a quasi $\bar{\sigma}$-rigid ring.

Recall that an element $u$ of a ring $R$ is right regular if $u r=0$ implies $r=0$ for $r \in R$. Similarly, left regular elements can be defined. An element is regular if it is both left and right regular (and hence not a zero divisor). A ring $R$ is called right (resp., left) Ore if given $a, b \in R$ with $b$ regular there exist $a_{1}, b_{1} \in R$ with $b_{1}$ regular such that $a b_{1}=b a_{1}$ (resp., $b_{1} a=a_{1} b$ ). It is a well-known fact that $R$ is a right (resp., left) Ore ring if and only if the classical right (resp., left) quotient ring of $R$ exists. 
Let $\sigma$ be an automorphism of a ring $R$. Suppose that there exists the classical right quotient ring $Q(R)$ of $R$. Then for any $a b^{-1} \in Q(R)$ where $a, b \in R$ with $b$ regular, the induced map $\bar{\sigma}: Q(R) \rightarrow Q(R)$ defined by $\bar{\sigma}\left(a b^{-1}\right)=\sigma(a) \sigma(b)^{-1}$ is also an automorphism. Note that the classical right quotient ring $Q(R)$ of a $\sigma$-rigid $\operatorname{ring} R$ is $\bar{\sigma}$-rigid. Similarly, we have the following.

Proposition 3.12. Suppose that there exists the classical right quotient ring $Q(R)$ of a ring $R$. If $R$ is a quasi $\sigma$-rigid ring, then $Q(R)$ is a quasi $\bar{\sigma}$-rigid ring.

Proof. Suppose that $a b^{-1} Q(R) \bar{\sigma}\left(a b^{-1}\right)=0$. Then $0=a b^{-1} Q(R) \bar{\sigma}\left(a b^{-1}\right)=$ $a Q(R) \sigma(a) \sigma(b)^{-1}$, since $b^{-1} Q(R)=Q(R)$. This implies $a Q(R) \sigma(a)=0$, and so $a R \sigma(a)=0$. Since $R$ is a quasi $\sigma$-rigid ring, we get $a=0$ and thus $Q(R)$ is a quasi $\bar{\sigma}$-rigid ring.

\section{Applications}

Recall that a ring $R$ is called Baer [15] if the right (left) annihilator of every nonempty subset of $R$ is generated by an idempotent; and a ring $R$ is called quasi-Baer [6] if the right annihilator of each right ideal of $R$ is generated (as a right ideal) by an idempotent. It is well-known that the (quasi-)Baerness of a ring is left-right symmetric. A ring $R$ is called a right (resp., left) p.p.-ring if the right (resp., left) annihilator of an element of $R$ is generated by an idempotent. $R$ is called a p.p.-ring if it is both a right and left p.p.-ring. From [4], a ring $R$ is called right (resp., left) principally quasi-Baer (or simply, right (resp., left) p.q.-Baer) if the right (resp., left) annihilator of a principal right (resp., left) ideal of $R$ is generated by an idempotent. $R$ is called a p.q.-Baer ring if it is both right and left p.q.-Baer. The class of p.q.-Baer rings includes all biregular rings, all (quasi-)Baer rings and all abelian (i.e., its idempotents are central) p.p.-rings. The extensions of Baer, quasi-Baer, p.q.-Baer and p.p.-rings have been studied by many authors $[2,4,6,7,8]$. In [9], it was proved that for a $\sigma$-rigid $\operatorname{ring} R$, a $\operatorname{ring} R$ is (quasi-)Baer if and only if $R[x ; \sigma, \delta]$ is a (quasi-)Baer ring if and only if $R[[x ; \sigma]]$ is a (quasi-)Baer ring; and $R$ is a p.q.-Baer (resp., p.p.-) ring if and only if $R[x ; \sigma, \delta]$ is a p.q.-Baer (resp., p.p.-) ring. Moreover, there exists a commutative von Neumann regular ring $R$ (and so a p.q.-Baer ring and a p.p.-ring), but $R[[x ; \sigma]]$ is neither a p.q.-Baer ring nor a p.p.-ring by $[9$, p. 225]. As parallel results to these, we have the following for a quasi $\sigma$-rigid ring.

Lemma 4.1. Let $R$ be a quasi $\sigma$-rigid ring.

(1) For any $p(x)$ and $q(x)$ in $R[x ; \sigma, \delta]$ (resp., $R[[x ; \sigma]]), p(x) R[x ; \sigma, \delta] q(x)=$ 0 (resp., $p(x) R[[x ; \sigma]] q(x)=0$ ) if and only if $a R b=0$ for all coefficients $a, b$ of $p(x)$ and $q(x)$, respectively.

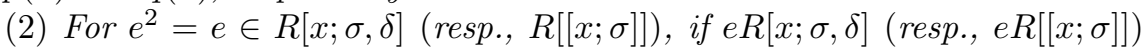

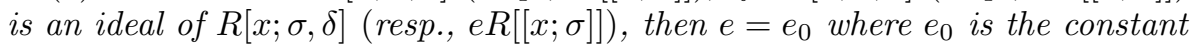
term of $e$. 
Proof. (1) It follows from Theorem 2.6. (2) Now $1-e=\left(1-e_{0}\right)-\sum_{i=1}^{n} e_{i} x^{i}$. Since $e R[x ; \sigma, \delta]$ is an ideal, we have $(1-e) R[x ; \sigma, \delta] e \subseteq(1-e) e R[x ; \sigma, \delta]=0$, and so $(1-e) R[x ; \sigma, \delta] e=0$. By (1), $\left(1-e_{0}\right) R e_{0}=0$ and $e_{i} R e_{i}=0$ for any $1 \leq i \leq n$. Since $R$ is semiprime by Lemma 2.1, we have $e_{i}=0$ for any $1 \leq i \leq n$. Therefore $e=e_{0}$.

The following example shows that the condition " $e R[x ; \sigma, \delta]$ is an ideal of $R[x ; \sigma, \delta]$ " in Lemma 4.1(2) cannot be dropped.

Example 4.2. Consider the quasi $\sigma$-rigid ring $R=\operatorname{Mat}_{2}(F)$ and the endomorphism $\sigma$ as in Example 1.1(1). Let $e=\left(\begin{array}{ll}0 & 1 \\ 0 & 1\end{array}\right)+\left(\begin{array}{ll}0 & 1 \\ 0 & 0\end{array}\right) x \in R[x ; \sigma]$. Then $e^{2}=e \in R[x ; \sigma]$ and $e \in e R[x ; \sigma]$. For $r=\left(\begin{array}{cc}1 & 0 \\ 0 & 0\end{array}\right) \in R[x ; \sigma]$, re $=$ $\left(\begin{array}{ll}0 & 1 \\ 0 & 0\end{array}\right)+\left(\begin{array}{ll}0 & 1 \\ 0 & 0\end{array}\right) x \notin e R[x ; \sigma]$, since the constant term of any element of $e R[x ; \sigma]$ is of the form $\left(\begin{array}{ll}a & b \\ a & b\end{array}\right)$ where $a, b \in F$. This implies that $e R[x ; \sigma]$ is not an ideal. Note that $e=\left(\begin{array}{ll}0 & 1 \\ 0 & 1\end{array}\right)+\left(\begin{array}{ll}0 & 1 \\ 0 & 0\end{array}\right) x \notin R$.

For a nonempty subset $S$ of a ring $R$, the right annihilator of $S$ in $R$ will be by $r_{R}(S)=\{c \in R \mid d c=0$ for any $d \in S\}$.

Proposition 4.3. Let $R$ be a quasi $\sigma$-rigid ring. The following are equivalent:

(1) $R$ is a quasi-Baer ring.

(2) $R[x ; \sigma, \delta]$ is a quasi-Baer ring.

(3) $R[[x ; \sigma]]$ is a quasi-Baer ring.

Proof. Without the assumption that $R$ is quasi $\sigma$-rigid, (1) $\Rightarrow(2)$ and $(1) \Rightarrow(3)$ were proved in [11, Theorem 1] and [5, Theorem 1.2] respectively. $(2) \Rightarrow(1)$ Assume that $R[x ; \sigma, \delta]$ is a quasi-Baer ring. Let $J$ be an ideal of $R$. Then $r_{R[x ; \sigma, \delta]}(J R[x ; \sigma, \delta])=e R[x ; \sigma, \delta]$ for some idempotent $e \in R$, by Lemma 4.1(2). Thus $r_{R}(J)=r_{R[x ; \sigma, \delta]}(J R[x ; \sigma, \delta]) \cap R=e R[x ; \sigma, \delta] \cap R=e R$ by Lemma $4.1(1)$. Therefore $R$ is a quasi-Baer ring. $(3) \Rightarrow(1)$ is also proved by the similar arguments above.

Observe that if $R$ is a quasi $\sigma$-rigid ring, then $R$ is a right p.q.-Baer ring if and only if $R$ is a left p.q.-Baer ring since $R$ is semiprime by [4, Corollary 1.11].

Theorem 4.4. Let $R$ be a quasi $\sigma$-rigid ring.

(1) $R$ is a right p.q.-Baer ring if and only if $R[x ; \sigma, \delta]$ is a right p.q.-Baer ring.

(2) If $R[[x ; \sigma]]$ is a right p.q.-Baer ring, then $R$ is a right p.q.-Baer ring.

Proof. (1) Assume that $R$ is a right p.q.-Baer ring. For any principal right ideal $I=p(x) R[x ; \sigma, \delta]$ of $R[x ; \sigma, \delta]$ where $p(x)=a_{0}+a_{1} x+\cdots+a_{m} x^{m}$, we take $I^{*}=a_{0} R+\cdots+a_{m} R$ as the finitely generated right ideal generated by $a_{0}, \ldots, a_{m}$. Since $R$ is right p.q.-Baer, $r_{R}\left(I^{*}\right)=e R$ for some $e^{2}=e \in R$. Note that $e$ is central since $R$ is semiprime. Then $I^{*} R e=0$, and so $p(x) R[x ; \sigma, \delta] e=$ 0 by Lemma 4.1(1). Hence, $I e=0$ and so $e \in r_{R[x ; \sigma, \delta]}(I)$. Thus $e R[x ; \sigma, \delta] \subseteq$ $r_{R[x ; \sigma, \delta]}(I)$. Now we let $q(x)=b_{0}+b_{1} x+\cdots+b_{n} x^{n} \in r_{R[x ; \sigma, \delta]}(I)$. Then $p(x) R[x ; \sigma, \delta] q(x)=0$ and thus $b_{0}, b_{1}, \ldots, b_{n} \in r_{R}\left(I^{*}\right)=e R$ by Lemma 4.1(1). 
Hence there exist $c_{0}, c_{1}, \ldots, c_{n}$ such that $q(x)=e c_{0}+e c_{1} x+\cdots+e c_{n} x^{n}=$ $e\left(c_{0}+c_{1} x+\cdots+c_{n} x^{n}\right) \in e R[x ; \sigma, \delta]$. Consequently, $r_{R[x ; \sigma, \delta]}(I)=e R[x ; \sigma, \delta]$. Therefore $R[x ; \sigma, \delta]$ is right p.q.-Baer. The proofs of the converses of both (1) and (2) follow the proof $(2) \Rightarrow(1)$ of Proposition 4.3 .

Remark 4.5. (1) The condition "quasi-Baer rings" in Proposition 4.3 can neither be replaced by "Baer rings" nor "right p.p.-rings": For example, let $R=\operatorname{Mat}_{2}(\mathbb{Z})$. Then $R$ is a Baer ring, but $R[x]$ is not right p.p. by $[9, \mathrm{Ex}-$ ample $10(2)]$. Also $R$ is a quasi $\sigma$-rigid ring, but $R[x ; \sigma]$ is neither Baer nor right p.p., in case $\sigma$ is the identity endomorphism of $R$.

(2) There exists a quasi $\sigma$-rigid and p.q.-Baer ring which is not quasi-Baer, letting $\sigma$ be the identity endomorphism of $R$ by [4, Lemma 1.4 and Example $1.5(\mathrm{i})]$

(3) The converse of Theorem 4.4(2) does not hold by [9, p. 225].

(4) The condition " $R$ is a quasi $\sigma$-rigid ring" in Proposition 4.3 and Theorem 4.4 is not superfluous by [9, Example 9].

From [9, Example 9], we see that there exists a semiprime ring $R$ with $\sigma(e)=e$ for any central idempotent $e \in R$ such that $R[x ; \sigma, \delta]$ is p.q.-Baer, but $R$ is not quasi $\sigma$-rigid. However, we have the following which is compared with Proposition 3.2.

Proposition 4.6. Let $R$ be a semiprime ring with $\sigma(e)=e$ for any central idempotent $e \in R$. If $R$ is a right p.q.-Baer ring, then $R$ is quasi $\sigma$-rigid.

Proof. Suppose that $R$ is right p.q.-Baer and $a R \sigma(a)=0$ for $a \in R$. Then $\sigma(a) \in r_{R}(a R)=e R=\sigma(e R)$ where $e=e^{2} \in R$ is central since $R$ is semiprime. It follows that $a \in e R$, entailing $a R a=0$ and hence $a=0$ since $R$ is semiprime. Therefore $R$ is quasi $\sigma$-rigid.

The condition " $\sigma(e)=e$ for any central idempotent $e \in R$ " in Proposition 4.6 cannot be dropped. For the ring $R=\mathbb{Z}_{2} \oplus \mathbb{Z}_{2}$ with an automorphism $\sigma$ in Example 1.1(2), $R$ is semiprime and right p.q.-Baer but not quasi $\sigma$-rigid and $\sigma(1,0) \neq(1,0)$.

\section{References}

[1] S. Annin, Associated primes over skew polynomial rings, Comm. Algebra 30 (2002), no. $5,2511-2528$

[2] M. Başer, A. Harmanci, and T. K. Kwak, Generalized semicommutative rings and their extensions, Bull. Korean Math. Soc. 45 (2008), no. 2, 285-297.

[3] G. F. Birkenmeier, H. E. Heatherly, and E. K. Lee, Completely prime ideals and associated radicals, Ring theory (Granville, OH, 1992), 102-129, World Sci. Publ., River Edge, NJ, 1993.

[4] G. F. Birkenmeier, J. Y. Kim, and J. K. Park, Principally quasi-Baer rings, Comm. Algebra 29 (2001), no. 2, 639-660.

[5] , Polynomial extensions of Baer and quasi-Baer rings, J. Pure Appl. Algebra 159 (2001), no. 1, 25-42. 
[6] W. E. Clark, Twisted matrix units semigroup algebras, Duke Math. J. 34 (1967), 417423.

[7] E. Hashemi, Compatible ideals and radicals of Ore extensions, New York J. Math. 12 (2006), 349-356.

[8] E. Hashemi and A. Moussavi, Polynomial extensions of quasi-Baer rings, Acta Math. Hungar. 107 (2005), no. 3, 207-224.

[9] C. Y. Hong, N. K. Kim, and T. K. Kwak, Ore extensions of Baer and p.p.-rings, J. Pure Appl. Algebra 151 (2000), no. 3, 215-226.

[10] _ On skew Armendariz rings, Comm. Algebra 31 (2003), no. 1, 103-122.

[11] C. Y. Hong, N. K. Kim, and Y. Lee, Ore extensions of quasi-Baer rings, Comm. Algebra 37 (2009), 2030-2039.

[12] C. Y. Hong and T. K. Kwak, On minimal strongly prime ideals, Comm. Algebra 28 (2000), no. 10, 4867-4878.

[13] C. Y. Hong, T. K. Kwak, and S. T. Rizvi, Rigid ideals and radicals of Ore extensions, Algebra Colloq. 12 (2005), no. 3, 399-412.

[14] A. A. M. Kamal, Some remarks on Ore extension rings, Comm. Algebra 22 (1994), no. 10, 3637-3667.

[15] I. Kaplansky, Rings of operators, W. A. Benjamin, Inc., New York-Amsterdam, 1968.

[16] N. K. Kim and T. K. Kwak, Minimal prime ideals in 2-primal rings, Math. Japon. 50 (1999), no. 3, 415-420.

[17] J. Krempa, Some examples of reduced rings, Algebra Colloq. 3 (1996), no. 4, 289-300.

[18] T. Y. Lam, A. Leroy, and J. Matczuk, Primeness, semiprimeness and prime radical of Ore extensions, Comm. Algebra 25 (1997), no. 8, 2459-2506.

[19] T. K. Lee and Y. Q. Zhou, Armendariz and reduced rings, Comm. Algebra 32 (2004), no. 6, 2287-2299

[20] G. Marks, On 2-primal Ore extensions, Comm. Algebra 29 (2001), no. 5, 2113-2123.

[21] G. Mason, Reflexive ideals, Comm. Algebra 9 (1981), no. 17, 1709-1724.

[22] K. R. Pearson and W. Stephenson, A skew polynomial ring over a Jacobson ring need not be a Jacobson ring, Comm. Algebra 5 (1977), no. 8, 783-794.

CHAN YONG HONG

Department of Mathematics and Research Institute for Basic Sciences

KyUng HeE UNIVERSITY

SEOUl 131-701, KoreA

E-mail address: hcy@khu.ac.kr

NAM KYUN KIM

Division of General Education

Hanbat National University

DAEJEON 305-719, KOREA

E-mail address: nkkim@hanbat.ac.kr

Tai Keun KwaK

Department of Mathematics

DAEJIN UNIVERSITY

PocheOn 487-711, KoreA

E-mail address: tkkwak@daejin.ac.kr 